\title{
Trends in Injection Opioid Use and Bloodborne Pathogen Related Diseases in New Jersey
}

\author{
Jenna Lifshitz 2,1 , Pinar Erdogdu², Stella Tsai ${ }^{2}$ \\ ${ }^{1}$ Brandeis University, Waltham, Massachusetts, United States, ${ }^{2}$ New Jersey Department of Health, Trenton, New Jersey, United States
}

Objective

To utilize New Jersey's syndromic surveillance data in the study and comparison of trends in injection opioid use and infection with selected bloodborne pathogens (BBPs) over the years 2013-2017.

\section{Introduction}

When the opioid epidemic began in the early 1990s, pills such as oxycodone were the primary means of abuse. Beginning in 2010 , injection use of, first, heroin and then synthetic opioids dramatically increased, which led the number of overdose deaths in volving opioids to increase fivefold between 1999 and 2016 [1]. It would be expected that BBP rates would rise with this increase in injection use, and, nationally, there has been a rise in acute hepatitis $\mathrm{C}(\mathrm{HCV})$ rates, although the other two main BBPs, acute hepatitis B (HBV) and acute human immunodeficiency virus (HIV) have been flat and declining, respectively [2,3].

In this study, we compared New Jersey's reported incidence of these three BBPs (acute HBV, acute HCV, and HIV) over five years (2013-2017) with syndromic surveillance data for opioid use over the same time period in order to test the hypothesis that emergency department (ED) visits for opioid use could be used as a predictor of BBP infection.

\section{Methods}

To indirectly track the number of injection opioid users, we wrote a custom classifier for EpiCenter, New Jersey's statewide syndromic surveillance system, to search ED chief complaints for the number of visits related to injection opioid usage. Our custom classifier creation started with the CDC's National Syndromic Surveillance Program (NSSP) ESSENCE Chief Complaint Category classifier for opioid overdose [4]. As we were looking to count not just overdoses but all visits likely to be associated with injection drug use, we chose not to omit the keyword "withdrawal," differing from CDC's classifier in which it is a negative indicator.

Positive indicator keywords included "opioid," "heroin," "narcot," “opiate," "fentanyl," "naloxo," "narcan," "ivdu," and the ICD9 and ICD-10 codes e850.[0-2], 304, 305.5, f11, t40.[0-6], and 965. These keywords were used to target the chief complaints of people using injection opioids. Negative indicators included "patch," "allerg," and "med" to eliminate medical opioid use. Negative indicators also included "vicodin," "tramadol," "percocet," "oral," and t40.5 to filter out opioids most commonly used in pill form, as well as other drugs.

Cases of acute HCV and acute HBV were totaled using CDRSS, New Jersey's Communicable Disease Reporting and Surveillance System. In order to maintain consistency, we used the respective 2012 case definition for each disease. Numbers of new HIV infections were accessed from NJ's reportable disease list [5]. All of the data sets followed the epidemiologic years 2013-2017 (based upon MMWR weeks).

\section{Results}

HIV diagnosis rates slightly decreased over time. HBV rates trend upwards, similar to the rates of injection drug use (IDU) for the first three years but start to drop after 2015. Aside from an unexplained dip in 2016, the HCV rates generally track the EpiCenter data for IDU (Figure 1). On a regional scale, NJ's Northwest region had the highest rates per capita of the five NJ regions and the most similar trending between the HCV and EpiCenter data sets (Figure 2). This result follows the nationwide trend of the opioid epidemic occurring more widely in rural areas, as this region is the most rural region in New Jersey [6].

In figures $1 \& 2$, IDU (EpiCenter) and HIV are plotted on the primary (left) axis and HCV and HBV are plotted on the secondary (right) axis.

ISDS Annual Conference Proceedings 2019. This is an Open Access article distributed under the terms of the Creative Commons AttributionNoncommercial 4.0 Unported License (http://creativecommons.org/licenses/by-nc/3.0/), permitting all non-commercial use, distribution, and reproduction in any medium, provided the original work is properly cited. 


\section{Conclusions}

Both IDU related visits and cases of acute HCV show an ongoing upward trend. This result agrees with the initial hypothesis. However, the association between acute HBV cases and IDU wasn't as strong. This finding can be attributed to the fact that while $\mathrm{HBV}$ is a BBP, it is most commonly transmitted vertically from an infected mother to her child at birth, whereas HCV is primarily transmitted through the sharing of needles or syringes [7,8]. There is no apparent relationship between HIV rates and injection drug rates, likely because HIV has a $0.3 \%$ infection risk rate from a single infected needlestick versus the $1.8 \%$ risk of acquiring $\mathrm{HCV}$ and $22-31 \%$ risk of acquiring HBV [9].

\section{Acknowledgement}

We would like to thank Bernice Carr, MS, MPH, Maryellen Wiggins, MSN, RN, Teresa Hamby, MSPH, and Abdel R. Ibrahim, Ph.D. for their contributions.

\section{References}

1. Centers for Disease Control and Prevention (CDC). Understanding the Epidemic; August 30, 2017. https://www.cdc.gov/drugoverdose/epidemic/index.html. Accessed 13 July 2018.

2. CDC. Viral Hepatitis; May 19, 2016. https://www.cdc.gov/hepatitis/hbv/statisticshbv.htm. Accessed 24 July 2018.

3. CDC. HIV in the United States: At A Glance; June 26, 2018.

https://www.cdc.gov/hiv/statistics/overview/ataglance.html. Accessed 24 July 2018.

4. CDC. NSSP Update; May, 2017. https://www.cdc.gov/nssp/documents/nssp-update-2017-05.pdf. Accessed 23 July 2018.

5. NJDOH. HIV, STD, and TB Services; December, 2016. https://www.cdc.gov/hiv/statistics/overview/ataglance.html Accessed 27 July 2018

6. Schranz AJ, Barrett J, Hurt CB, Malvestutto C, Miller WC. 2018. Challenges Facing a Rural Opioid Epidemic: Treatment and Prevention of HIV and Hepatitis C. Curr HIV/AIDS Rep. 15(3), 245-54. PubMed https://doi.org/10.1007/s11904-018-0393-0

7. Rolls DA, et al. 2013. Hepatitis C Transmission and Treatment in Contact Networks of People Who Inject Drugs. PLoS One. 8(11), e78286. PubMed https://doi.org/10.1371/journal.pone.0078286

8. Perrillo RP. 1993. Hepatitis B: transmission and natural history. Gut. 34(2 Suppl), S48-49. PubMed https://doi.org/10.1136/gut.34.2_Suppl.S48

9. Berry AJ. 2004. Needle stick and other safety issues. Anesthesiol Clin North America. 22(3), 493-508. PubMed https://doi.org/10.1016/j.atc.2004.04.003 


\section{ISDS 2019 Conference Abstracts}

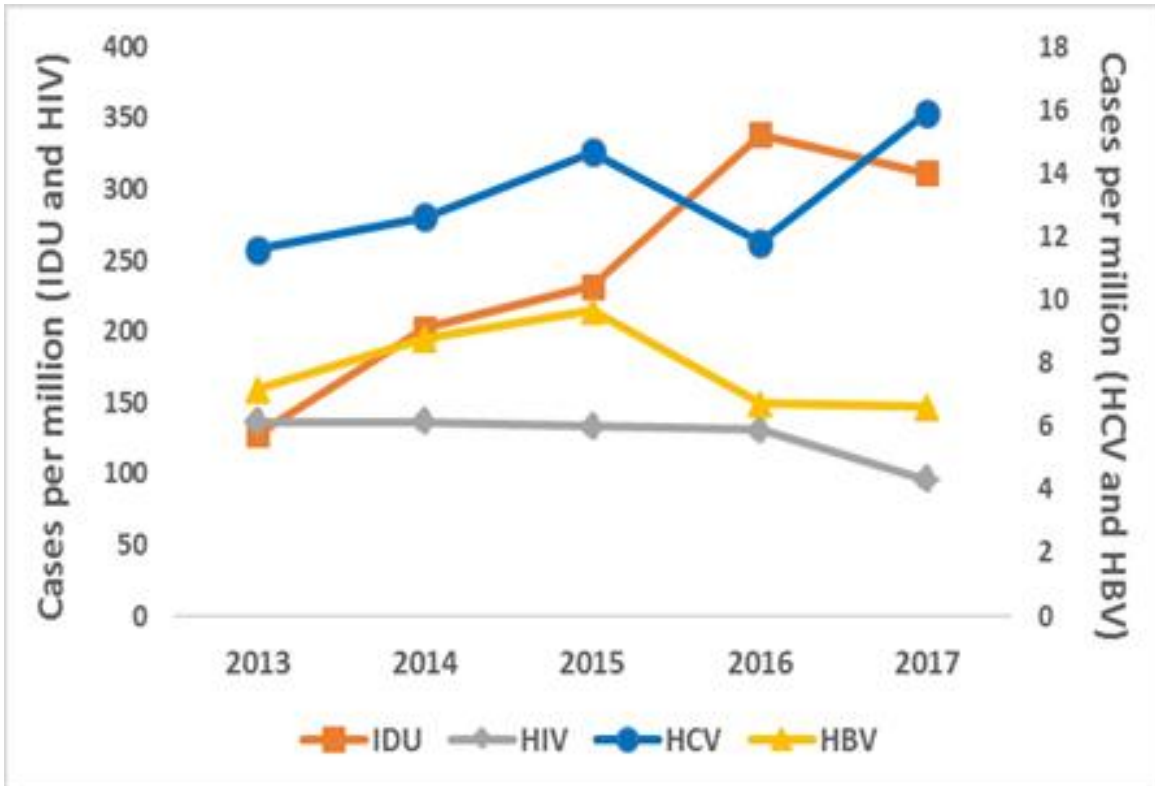

Figure 1: Comparison of HCV, HBV, HIV, and IDU (measured by EpiCenter in ED Visits related to Injection Drug Use) data for all of NJ in cases per million for 2013-2017

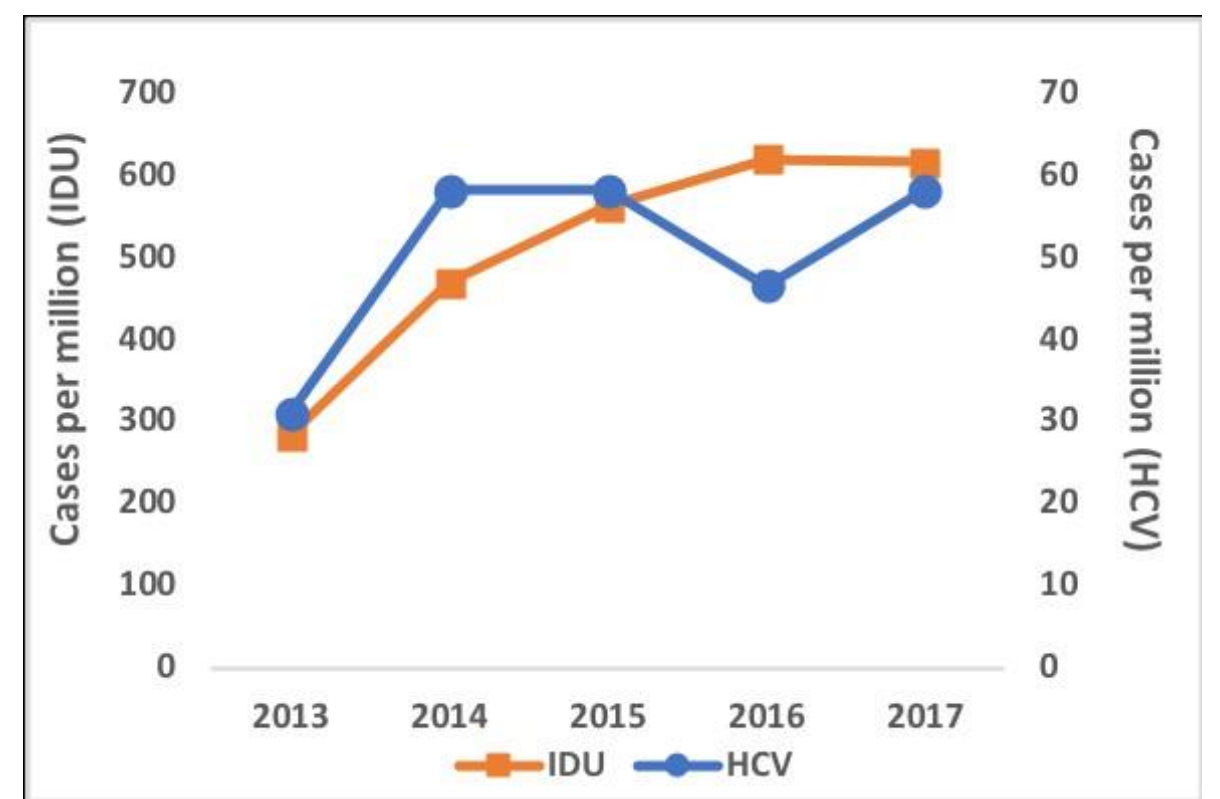

Figure 2: Comparison of HCV and IDU (measured by EpiCenter in ED Visits related to Injection Drug Use) data for the Northwest NJ Region (Morris, Passaic, Sussex, and Warren counties) in cases per million for 2013-2017 